\title{
Handwritten Character Recognition from Ancient Palm Leaves using Gabor based MultiLayer Architecture:GMA
}

\author{
Jyothi.R.L ${ }^{1}$ and Abdul Rahiman.M ${ }^{2}$ \\ ${ }^{1}$ University of Kerala and College of Engineering Chengannur, Kerala, India. \\ ${ }^{2}$ University of Kerala and LBS Institute of Technology for Women, Kerala, India.
}

\begin{abstract}
Feature extraction plays the key role in pattern recognition systems. With the invent of Deep learning algorithms it is believed that the importance of feature extraction methods has been reduced. But the cost of implementing deep algorithms is very high. Deep neural networks rely on GPU architecture and requires large amount of data for achieving high recognition efficiency. It is computationally expensive to train the deep architecture and more over the learning procedure and factors for training is not easy to realize. Therefore having inspired by the structure of Deep Convolutional Neural Network a new feature extraction method based on conventional feature extraction system for recognition is proposed. In this method a multilayer architecture is designed with convolution layer based on gabor filter and classification layer based on Artificial Neural Network.Only the classification layer is subjected to learning by backpropogation and all other layers acts as the part of feature extraction system. The input image without applying any pre-processing can be subjected to the proposed system which in turn predicts the class of image as output. The proposed method is compared with some of the existing efficient feature extraction methods like discrete meyer wavelet, zernike moment, curvelet, legendre moments, gaussian hermite $(\mathrm{GH})$ moment and Histogram of gradient(HOG). The recognition efficiency produced by the method without applying any pre-processing on input images is much higher than existing efficient feature extraction methods with preprocessing applied.The proposed method works effectively invariant to noise, translation and rotation. Experimental analyses were carried out in two datasets. First dataset is the standard HPL dataset of isolated Tamil characters. The second dataset consists of Grantha characters extracted from ancient palm leaves.
\end{abstract}

Keywords: Gabor based multilayer architecture Grantha dataset HPL dataset small-sized dataset scale invariant translation invariantrotation invariant palm leaves

\section{INTRODUCTION}

Handwritten character recognition is a prominent research area. Due to the large variation seen in the handwritings from one user to another, handwritten character recognition systems have not yet reached a satisfactory recognition level. Different steps in character recognition are pre-processing, feature extraction and classification. Here the most important step is the feature extraction. The efficiency of a recognition system can be increased by selecting an apt feature extraction method. With the discovery of deep learning concept the importance of feature extraction stage has been decreased. But for the working of deep architecture [1] GPU framework is essential otherwise it will take several days to get a result with the CPU frame work. Therefore to enjoy the complete benefit from deep architecture, high initial cost is needed or should depend upon some high end cloud platform, which makes the task more complex. Moreover there is a need of large dataset domain for the working of deep architecture which is not always possible. Even though transfer learning based CNN solves the problem of limited size dataset, the performance of transfer network[2] depreciates with the increase in distance between base dataset (dataset using which the transfer layers are learned) and target dataset (new dataset used for learning)[2]. Hence this work concentrates on conventional feature extraction style. Here a new method has been devised by taking into account the structure of CNN framework. The image is passed through different layers before the final feature extraction stage. The convolution layer which is one of the important layers in CNN (Convolutional Neural Network) type deep learning architecture is designed here based on Gabor filter. The main difference between CNN and proposed work is that entire set of layers in $\mathrm{CNN}$ is subjected to relearning through back propagation [3] but the proposed work is a simple conventional feature extraction method with relearning performed only in the classification layer. The proposed work is compared with some of the existing efficient feature extraction methods and it is found that this newly devised method gives better recognition efficiency compared to other methods.

The domains chosen for training and testing the recognition system are ancient grantha script [4] and standard HPL dataset consisting of isolated Tamil characters. Grantha script has been chosen in this work, as there exist only few works reported for its recognition. Grantha script is the script used in southern part of India to write Sanskrit language. Sanskrit language was considered as the most royal language in ancient India. Ancient literatures and valuable documents in India were written in Sanskrit language. The documents written in grantha script now exist in the form of palm leaves, stone inscriptions and copper plates. This work concentrates on recognition of grantha characters taken from ancient palm leaves. A part of a palm leaf consisting of grantha characters is shown in Fig.1. 


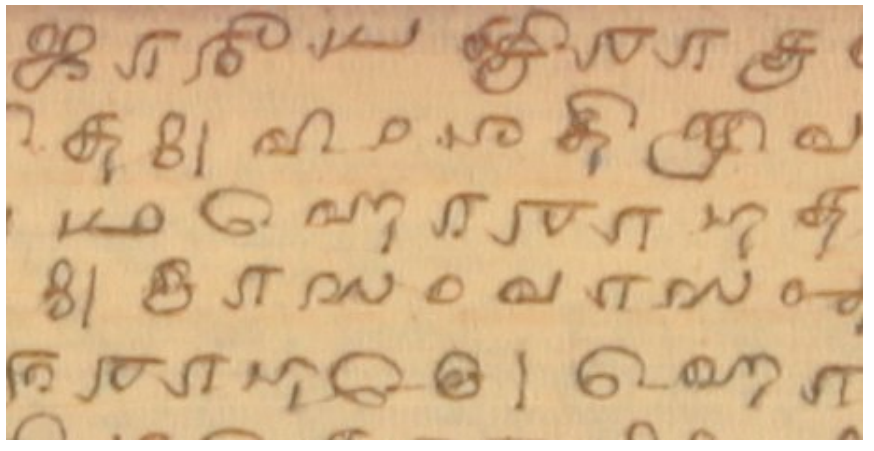

Figure 1: Grantha PalmLeaf

There are uncountable number of palm leaves exists in grantha script which can be found in various Oriental Research Institutes and archaeological libraries in different parts of the country. These palm leaves are in a state of decay. Only a few experts knew to read this script and if an efficient recognition system is built for the script we may able to protect valuable information that will be useful for the society. This work concentrates on palm leaves of grantha script taken from Oriental research Institute in Kerala, a small state in India. Grantha script consists of 16 vowels(out of which 7 are basic symbols), 34 consonants and 9 numerals. There also exist complex consonantal clusters in Grantha script formed in combination of special signs. Fig.2 shows grantha characters and numerals.

The second dataset is the standard HPL dataset of isolated Tamil characters. The images in this character data set are binary and almost noise free.

Section 2 depicts the literature survey. Gabor based multilayer architecture is explained in section 3. Experimental analysis is depicted in section 4 and conclusion in section 5 .

\section{LITERATURE SURVEY}

The main part of developing an efficient character recognition system is to identify apt features which may lead to fewer recognition errors. Statistical learning theory which is a method of comparing different feature extraction algorithms cannot be directly compared with deep architecture directly. Deep learning is a machine learning framework [1],[5]. Eventhough deep architecture gives better recognition compared to other solutions in multiple domains and reduce the need of feature extraction, have several disadvantages. One of the main disadvantage is that it requires thousands of data samples for training and will give poor performance in limited sample domain. Second disadvantage is that deep framework is highly computationally expensive. The most complex deep model may take weeks to train using hundreds of machines equipped with expensive GPUs. Determining the topology, training method and hyper parameters for deep learning is like a black box with no theory to guide. Moreover what is learned by deep framework is not easy to comprehend like other classifiers.

Feature extraction is mainly classified into structural feature extraction, statistical
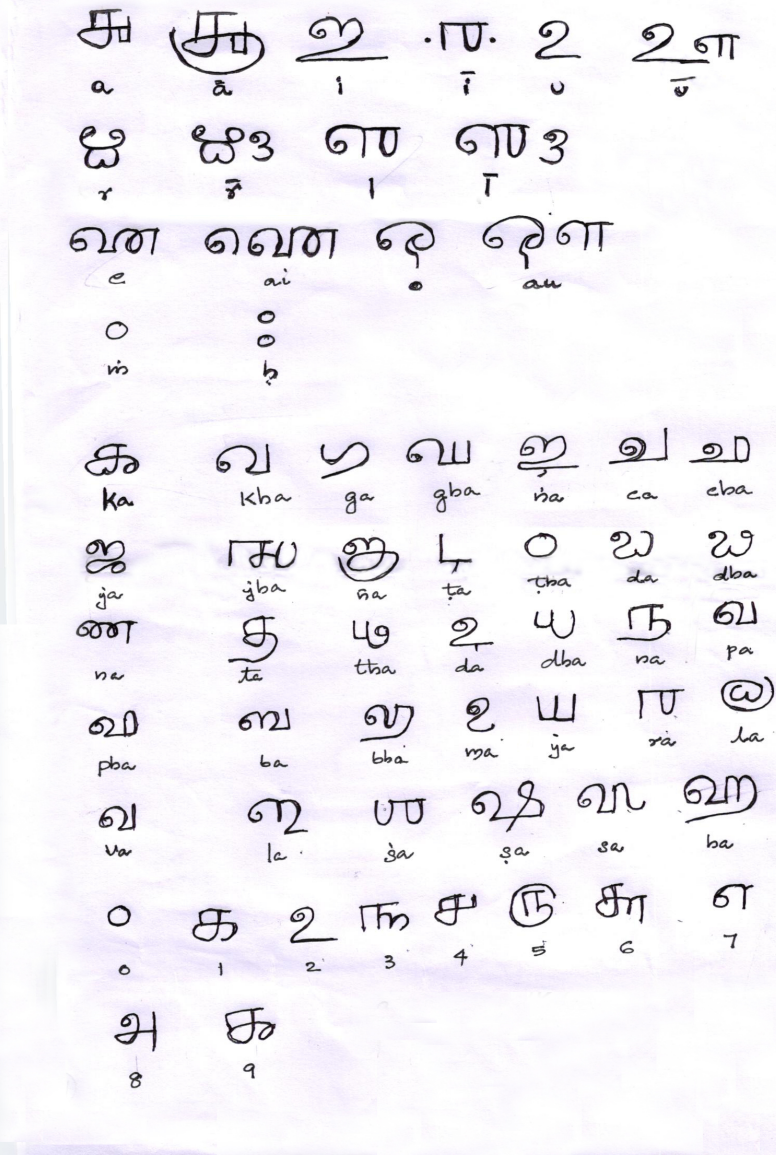

Figure 2: Grantha Characters

feature extraction and global transformation[6]. Structural features include dots and positions, strokes, width and height of strokes, directions and intersections of line segments and loops[7]. Statistical feature extraction methods include spatial distribution of pixels, zoning and histogram of chain codes. Global transformations include Fourier transform, Discrete Cosine transform, wavelets, hough transform and moments[8],[9],[10].

To build an efficient object recognition system, the feature extraction methods should be robust towards noise, distortion, style variation' translation and rotation. Structural feature extraction methods are less complex but works very low in noise and medium in rotation, translation and style variation. Statistical feature extraction methods works medium in distortion and rotation but works very low in noise, style variation and translation. Global transformation based methods are very complex but works high in distortion, style variation, translation and rotation when compared to structural and statistical feature extraction methods. All the above mentioned classes of feature extraction methods works very low in noise[11] Global transform based feature extraction methods works invariant to translation, rotation, style variation and distortion compared to other feature extraction methods. In [12] a comparison of geometric, central and Lengendre moments were carried out and it has been concluded that Lengendre moments works better compared to geometric 
moments. The experiments were carried out in 6763 chinese characters defined in China's national standard GB2312.In [12] the authors also concluded that moments are global features that does not produce good recognition results when used alone and should be used in conjunction with other methods to build an efficient recognition system.

$\operatorname{In}[13]$ a new set of orthogonal complex moments known as Zernike moments were introduced and compared with regular and moment invariants. Here experiments were carried out in two different dataset. First set is a character dataset with each character of 12 different orientations and image size of 64 by 64 .The second dataset is 12 differently oriented images of lakes Eric, Huron, Michigan and Superior with image size of 64 by 64 . Here it is concluded that Zernike moments works better compared to other moments. Moreover the authors in this work assert that Zernike moments are only rotation invariant and to make it scale and translation invariant it should be used in conjunction with regular moments. For classification minimum distance and nearest neighbor classifier is used.The experiments were carried out in 3 different types of signal to noise ratio $30 \mathrm{~dB}, 25 \mathrm{~dB}$ and $17 \mathrm{~dB}$ and better performance is achieved when the signal to noise ratio is $25 \mathrm{~dB}$.

In[14] a comparison of lengendre, Zernike and Pseudo Zernike moments for feature extraction of iris recognition is carried out. The numerical results indicate that the recognition rate of all the moments are identical but in noisy images lengendre moments works better compared to other moments. In $\operatorname{In}[15]$ a comparison of geometric, Zernike and pseudo zernike moments were carried out and concluded that Zernike moments works better compared to other moments in the presence of white noise. Here the performance comparison is carried out in the CASIA dataset. In [16] a comparison of $\mathrm{Hu}$, Zernike, Legendre moments and a newly devised method cumulants were carried out. Cumulants are quantities similar to moments which is the Taylor series expansion of cumulant generating function. Here comparison of $\mathrm{K}$ nearest neighbour(KNN) and Support Vector Machine(SVM) classifiers have been carried out. Legendre moments outperform other moments when features are classified using both KNN and SVM. Using SVM the recognition rate of Legendre moment is 95 percentage but using $\mathrm{KNN}$ the recognition rate is 85 percentage. In this work it has been asserted that cumulants are highly sensitive to noise compared to other methods and produce lower recognition rate compared to other methods. In[17] comparison between wavelet moment invariants with Bspline and Zernike moments were carried out.The testing and training were carried out in two set of data. First set consisting of two artificially generated shapes consisting of 60 images in which 30 from each shape and testing is carried out with 100 images 50 from each shape The second set consist of 26 uppercase English letters in which 30 randomly generated images each were selected for training and testing. Here wavelet moment invariants with Bspline provide higher recognition efficiency compared to other moment methods. In[18] a method for object recognition is implemented using wavelet based salient points. Here features are extracted using basic moments from the image after performing wavelet transform. The authors claim that the method has high recognition rate compared to other methods.In [19] the concept of Gaussian Hermite moments (GH moments) was described.GH moments are robust with respect to noise compared to other moments. The Guassian Hermite moment is the only moment that is orthogonal to rectangular area which offer a possibility of an efficient design of rotation invariant moments suitable for digital images. The main disadvantage of Gaussin Hermite moment is that, it is not scale invariant. In [19] a method has been proposed to make GH moment invariant to scaling. Zernike moments and other radial moments are not inherently invariant to scaling. But the images are mapped to unit disk to make the moment scale invariant. In [19] authors claim that the numerical accuracy of Zernike moments are less compared to GH moment. Moreover Zernike moments introduce more error compared to GH moment. In [20] features extracted from different types of wavelet transforms like daubachies, biorthogonal, reverse biorthogonal, Symlet, Coiflet, casual and generalized fractional Bsplines are compared using KNN classifier and found that recognition efficiency of above 91 percentage is given by daubachies, Biorthogonal, reverse biorthogonal, casual and generalized fractional BSplines.

In[21] a comparative analysis of wavelet, curvelet and gabor transform are carried out for face recognition and it is found that curvelet works better compared to others. On comparing wavelet and gabor, gabor works better on taking into account illumination changes of images. In[22] a Comparative study in wavelet, curvelet and Contourlet is carried out. In this work the authors have asserted that in higher resolution wavelet acts as good features .But as resolution is decreased curvelets works better than wavelets.In lower and higher resolution contourlet has lower efficiency compared to wavelet and curvelet. In [23] a comparative analysis of different wavelet transform were carried out and found that Discrete meyer wavelet transform produces higher recognition compared to other wavelet transforms. Here domain that has been chosen for recognition is grantha characters extracted from palm leaves.

Based on literature analysis it can be asserted that global transform based feature extraction is better compared to structural and statistical feature extraction. Moments are global transformation features which cannot detect minute change variation. Therefore an efficient object recognition system can be built by combining wavelets and moments. In case of moments it can be seen that Legendre moments and Gaussian Hermite moments are better compared to Zernike and pseudozernike moments. But Zernike moments are better than Pseudozernike, $\mathrm{Hu}$ and Geometrical moments. But when wavelets and moments are compared, performance of the methods chosen depends upon the domain chosen. But if moments and wavelets are combined the efficiency of recognition system can be increased. When gabor, wavelet, curvelet and contourlet are compared contourlet produces poor recognition efficiency but the performance of gabor, wavelet and contourlet may vary depending upon the training and testing domain. Since gabor transform produces an outstanding performance when used in building a recognition 
system, a gabor based multilayer system is developed .A multilayer recognition system is developed here by taking into account the structure of Convolutional Neural Network.

\section{GMA: GABOR BASED MULTILAYER ARCHITECTURE}

The frame work of GMA architecture (Fig.3) is based on 4 layers created by convolution and maxpooling operation.

\subsection{Multiscale Layer}

In this layer the input image is scaled down into 12 scales and a pyramid of height 12 is created. This has been done to make architecture of GMA scale invariant. The pyramid of 12 scales are created using the equation

$$
\text { Pyramid }_{k}=\left[\left[\text { Image }_{\text {wcurrent }_{i k}, \text { lcurrent }_{i k}}\right]_{i=1}^{12}\right.
$$

where Pyramid $k$ is the pyramid of height '12' with scale reduction ratio [k:k].'wcurrent ${ }_{i k}$ ' is the current width of the pyramid scale with scale reduction value $\mathrm{k}$ and 'lcurrent ${ }_{i k}$ ' is the current length of the pyramid scale with scale reduction value k.Here 'i' which denote height of pyramid ranges between 1 to 12 and $\mathrm{k}$ (scale reduction value )ranges between 0 to 1 .The values for wcurrent $_{i k}$ and lcurrent $_{i k}$ for the $i^{\text {th }}$ scale of the pyramid is calculated from wprevious $_{i k}$ (previous width of the pyramid scale with scale reduction ratio $\mathrm{k}$ ) and lprevious $_{i k}$ (previous lenght of the pyramid scale with scale reduction ratio $\mathrm{k}$ ) using the given equation.

$$
\begin{array}{r}
\text { wcurrent }_{i k}=\text { wprevious }_{i k} / k \\
\text { lcurrent }_{i k}=\text { lprevious }_{i k} / k
\end{array}
$$

\subsection{Convolution Layer}

The previous layer output image at each scale reduction value $\mathrm{k}$ is convolved with a convolution mask.A convolution mask of size $11 * 13$ is designed based on gabor filter coefficients. The size of mask is chosen based on trial and error method. When the size of mask is increased the classification efficiency of the system is reduced and the working time complexity of the entire system is increased. Here orientation filters (convolution mask) for 17 orientations are created. These orientation filters make the proposed system rotation invariant. Since handwritten character images are subjected to slight change in orientation depending upon the writer, filters are designed in such a way it will take into account even the slightest orientation. Gabor filter coefficients are calculated as

$$
G(x, y)=\exp \left(\left(-\frac{\left(M^{2}+\gamma^{2} N^{2}\right)}{2 \sigma^{2}}\right) \cos \left(\frac{2 \pi}{\lambda} M\right)\right)
$$

Here $M=x \cos \theta-y \sin \theta$ and $N=x \cos \theta+y \sin \theta$ Here $\mathrm{x}$ varies from -5 to +5 and $y$ varies from -6 to +6 . The parameters $\gamma$ (aspect ratio) is set to 0.3 and $\sigma$ (effective width) is set to 4.1 and $\lambda$ (wavelength) is set to 5.2. Gabor filter of 17 orientations are created where $\theta$ varies from 0 to $\pi$. The value of $\theta$ for next iteration mask is calculated based on the relation new $\theta=$ old $\theta+11$.Using this relation the calculated $\theta$ values are $0,11, \ldots \ldots . .176$.The last value of $\theta$ is taken as 180 . Selection of various parameters in convolution layer Gabor filters are carried out based on experimental analysis which is explained in Section 4.Finally components of each orientation filter is normalized such that the mean is 0 and sum of their squares is 1.The output of a group of $\mathrm{M}$ image pixels is given by

$$
O(Y, G)=\left|\frac{\Sigma y_{i} g_{i}}{\sqrt{\Sigma y_{i}^{2}}}\right|
$$

where $y_{i}$ and $g_{i}$ are image pixel Coefficients and convolution filter coefficients. Each orientation filter is applied to all the 12 scales of image from the previous layer. After the application of orientation filters, 17 pyramids of size $X^{*} Y^{*} 12$ are created where $X$ ranges from 818 to 35 and $Y$ from 910 to 39 while moving from one scale of pyramid to another.

\subsection{Max pooling Layer}

A $10 * 10 * 2$ maxpooling mask is moved through the 17 pyramids (set of 17 pyramids created for each scale reduction value ' $k$ ')at a translation distance (stride)of 5 pixels. The resultant pyramid obtained after the operation is height reduced to half of the previous height of the pyramid. In this way the height of image pyramid is reduced to 6 from 12. Max pooling operation may also result in reduction of dimension in each scale. Dimension of the lower scale in each pyramid is reduced to $160 * 180$ and the dimension of upper scale in each pyramid is reduced to $6 * 8$. In the same way dimension at each scale is reduced. This layer serves for dimensionality reduction. Maxpooling operation is a subsampling process where the number of features for classification process has been considerably reduced.

\subsection{Sampling Layer}

A $3 * 4$ mask is moved through each scale of the pyramid. At the lowest scale the mask moves at a sampling distance of 6 in each pyramid and create the first feature vector comprising of features from the lower scale of all pyramids and at the next highest scale the mask moves at sampling distance 4 and in the next highest scale at a sampling distance of 3, then 2 and finally at a distance of 1 . At the last scale features are extracted without any sampling distance.

\subsection{Optimized Image Layer}

In this layer the feature matrix produced in previous layer is converted into single column matrix after eliminating all zeros. Elimination is carried out in such a way that the zeros are only eliminated if the count of simultaneous zero in the column of matrix is greater than a particular threshold. In this case the threshold for simultaneous zero count is taken as 3. Depending upon the dataset domain chosen for recognition the threshold value can be changed. 


\subsection{Classification layer}

The final feature vector obtained after the processing in previous layers is used for training and testing the classifier. Classification can be carried out using any of the existing classifier. In this work feed-forward neural network with 150 hidden neurons are used for training and testing.

\section{EXPERIMENTAL ANALYSIS}

\subsection{Selection of parameters of GMA Layers}

In Multiscale Layer a pyramid of height 12 scales are formed by resizing the images into various scales. This layer makes the proposed system invariant to scaling. Assuming that minimum size of a character image to be $10 \times 10$ and maximum size to be $1000 \times 1000$, the length and width of lowest layer scale is selected by iteration of length and width parameter from 10 to 1000. At each iteration of length and width a pyramid of height 20 (20 scales) is created. Ratio of reduction in length and width of the image at each scale (scale reduction ratio)is selected by checking the efficiency the system with scale reduction ratio values ranging from 0 to $1(0,0.1,0.2, \ldots .1)$. It is found that efficiency is high when scale reduction ratio is 0.7 . The experiment for finding the apt value of scale reduction ratio is repeated for scale reduction ratios between 0.60 to 0.80 and is found that highest recognition efficiency is produced when scale reduction ratio is $[0.77,0.77]$. Now height of the pyramid is selected by reducing the height of pyramid from 20. It is found that the system produces highest efficiency when height is 12. Pyramid height below and above 12 produces lower recognition efficiency. After iteration with different values of length and width, the maximum recognition efficiency is obtained when length and width of the lowest scale of the pyramid is 818 and 910 .

The size of the Gabor filter in convolution layer is also designed based on experimental analysis. Efficiency of the system is tested with Gabor filters of different size. The system is tested with Gabor filters of size $3 \times 3,5 \times 5$ and $7 \times 7$ and $11 \times 11$. Highest efficiency has been produced when the kernel size is $11 x 11$. In character images of Grantha and Tamil dataset as the width of image is greater than height, the test is repeated for kernels of size $11 \times 12,11 \times 13,11 \times 14$ and $11 \times 15$. The kernel of size $11 \times 13$ produces the highest recognition efficiency. In the same way kernels of max pooling layer and sampling layer were estimation by testing with different size kernels. The selection of $\sigma$ (effective width), $\gamma($ aspect ratio) and $\lambda$ (wavelength) is also carried out based on experimental analysis. It can be found that when $\sigma$ is $4.1, \lambda$ is 5.2 and $\gamma$ is 0.3 the system produces the highest recognition efficiency. Combinations of different values of $\lambda$ and $\sigma$ ranging between 1 and 10 is tested for the system and it is found that $\sigma$ values between 4 and 5 and $\lambda$ values between 5 to 6 produces highest recognition rate. Again testing of the recognition system is repeated with different combinations of $\gamma$ values between 4.0 to 5.0 and $\lambda$ between 5.0 to 6.0 and it is found highest efficiency of GMA is reported when $\sigma$ is 4.1 and $\lambda$ is 5.2. Fixing $\sigma$ as 4.1 and $\lambda$ as 5.2 different $\gamma$ values ranging from 0 to 0.5 is tested for GMA and it is found that fixing $\gamma$ values to 0.3 will increase the efficiency of the system.For selection of parameters the experiment is carried out in HPL dataset and Grantha dataset.Experimental analysis for selection of parameters of GMA layers were carried out by selecting 3 classes of 100 samples from each dataset.

\subsection{Evalution of GMA on HPL and Grantha Dataset}

The performance of GMA based feature analysis were evaluated using two data set. First dataset is the newly created data set of Grantha characters extracted from palm leaves. These Palm leaves were obtained from Oriental research Institute in Kerala (a small state in the Southern part of India). The second dataset is the standard HPL dataset of isolated Tamil Characters. HPL dataset is selected because Tamil characters have high similarity towards Grantha characters. Fig.4 depicts some of the isolated Grantha characters extracted from palm leaves and Tamil characters taken from HPL dataset.

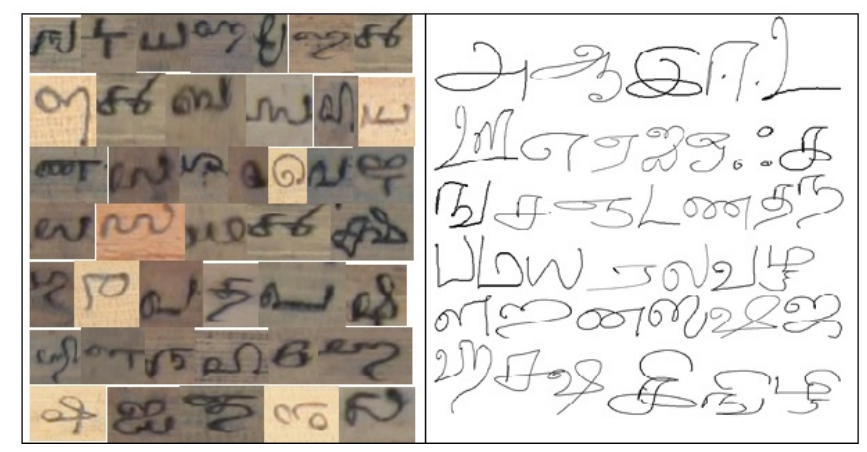

Figure 4: Left:Grantha Dataset, Right:Standard Tamil HPL Dataset

Grantha characters were extracted from 4015 folios of ancient palm leaves. The palm leaves used in this work are scanned with resolution 400 dpi. 41 Grantha characters of 290 samples each were extracted from the palm leaves. Sufficient samples of only 41 characters were obtained from palm leaves therefore only 41 characters out of the 50 Grantha characters were used in this work.

In case of standard HPL dataset 576 samples of each isolated characters are available. Out of which 288 character samples were used for training and other set of 288 character samples were for testing. The Tamil characters in HPL dataset are binary images and almost noise free. Therefore the performance of feature extraction method can be correctly evaluated using HPL dataset.

Comparative analysis of newly proposed method with wavelet transform, Curvelet, Gabor filter, Legendre moments, Zernike, Gaussian Hermite moment, Histogram of Oriented Gradient (HOG)[24]were carried out in both the data set. In case of GMA no preprocessing is applied for recognition of both datasets but in case of other methods Grantha images are binarized using sauvola method before applying the concerned algorithm. The feature vectors generated as the result 
of applying different feature extraction methods were fed to ANN classifier for classification. Feed forward neural network with 150 hidden neurons were trained with the feature vectors corresponding to each character. Table.1 shows the comparison of different feature extraction methods in Grantha and HPL data set .The above said methods were selected for comparison with GMA based feature extraction method because based on literature analysis it has been found that these methods produces higher object recognition efficiency.

Table 1: Recognition Efficiency of various Feature Extraction Methods

\begin{tabular}{lll}
\hline Method & Grantha Dataset & HPL dataset \\
\hline GMA & 91.63 & 94.4 \\
Curvelet & 76 & 79.2 \\
Dmeyer wavelet & 71 & 77.8 \\
Zernike moment & 74.3 & 78.3 \\
Legendre moment & 73.2 & 77.9 \\
GH moment & 73.4 & 76.4 \\
HOG & 85.8 & 94.3 \\
\hline
\end{tabular}

From the tabulated result it can be found that GMA based feature extraction method produces higher recognition efficiency. Fig. 5 depict the comparative analysis of recognition efficiency of various methods in Grantha Script and HPL dataset. Moreover it can be analysed that HOG produces higher recognition efficiency in case of HPL dataset but in case of Grantha dataset set the efficiency is much lower. In case of Grantha dataset the character images are highly noisy as they are extracted from palm leaves which are degraded due to aging but in case of HPL dataset the images are almost noise free binary images. Therefore it can be concluded that GMA based feature extraction method works efficiently even when the input image is degraded with noise.

The efficiency of feature extraction methods and classifier is tested by dividing the entire dataset into sample set for training,testing and validation .Here bootstrap based method is used for dividing the entire dataset into different samples. When the available dataset are not in thousands, it is better to use bootstrap method of data sample selection compared to holdout and crossover method. First a random sample set of 50 samples are chosen for each Grantha character. Out of which 10 samples are used for testing and 40 samples are used for training. Testing and training is repeated with the same sample set but with another 10 samples for testing and another set of 40 samples for training. In this way the experiment is repeated 5 times for a single sample set. The next sample set is selected by replacing first set of 10 samples with another set of 10 character samples from the dataset. In this way recognition system is analysed by training and testing with $29 * 5 * 5$ sample sets created from the given dataset. The results were analysed based on confusion matrices produced. Measures like accuracy, sensitivity, specificity, precision, F-measure and AUC were determined from the confusion matrices generated. For each of the 41 characters these measures were evaluated. The average values for accuracy, sensitivity, specificity, precision-measure and AUC for the Grantha dataset using GMA based method are shown in the Table 2. From the analyzed result it can be concluded that features produced based on Gabor based multilayer Architecture produces higher recognition efficiency compared to other methods.

Table 2: Analysis of various parameters based on Classification result produced using GMA and ANN in Ancient Grantha characters

\begin{tabular}{|c|c|c|c|c|c|c|}
\hline Character & Sensitivty & Specificity & Precision & Accuracy & F-measure & AUC \\
\hline $\mathrm{a}$ & 0.93 & 0.89 & 0.95 & 0.90 & 0.93 & 0.91 \\
\hline aah & 0.93 & 0.91 & 0.95 & 0.92 & 0.93 & 0.92 \\
\hline ba & 0.96 & 0.94 & 0.93 & 0.94 & 0.97 & 0.95 \\
\hline bha & 0.90 & 0.88 & 0.91 & 0.82 & 0.90 & 0.92 \\
\hline bla & 0.93 & 0.91 & 0.94 & 0.93 & 0.93 & 0.94 \\
\hline ccha & 0.98 & 0.95 & 0.99 & 0.96 & 0.98 & 0.98 \\
\hline chaa & 0.94 & 0.92 & 0.97 & 0.93 & 0.95 & 0.96 \\
\hline da & 0.98 & 0.94 & 0.90 & 0.94 & 0.98 & 0.99 \\
\hline daa & 0.93 & 0.91 & 0.95 & 0.92 & 0.93 & 0.92 \\
\hline dda & 0.92 & 0.94 & 0.94 & 0.94 & 0.93 & 0.92 \\
\hline fa & 0.88 & 0.86 & 0.91 & 0.87 & 0.89 & 0.90 \\
\hline gha & 0.98 & 0.94 & 0.95 & 0.95 & 0.99 & 0.98 \\
\hline gya & 0.94 & 0.93 & 0.95 & 0.94 & 0.94 & 0.9 \\
\hline haa & 0.93 & 0.90 & 0.95 & 0.91 & 0.94 & 0.92 \\
\hline $\mathrm{i}$ & 0.94 & 0.92 & 0.96 & 0.91 & 0.95 & 0.94 \\
\hline illa & 0.88 & 0.88 & 0.89 & 0.87 & 0.88 & 0.87 \\
\hline isha & 0.93 & 0.91 & 0.96 & 0.92 & 0.94 & 0.93 \\
\hline itha & 0.94 & 0.93 & 0.95 & 0.93 & 0.94 & 0.93 \\
\hline ithaa & 0.88 & 0.86 & 0.90 & 0.86 & 0.89 & 0.87 \\
\hline ja & 0.88 & 0.85 & 0.90 & 0.86 & 0.89 & 0.87 \\
\hline $\mathrm{ka}$ & 0.88 & 0.86 & 0.89 & 0.87 & 0.88 & 0.87 \\
\hline laa & 0.94 & 0.92 & 0.95 & 0.92 & 0.94 & 0.93 \\
\hline leya & 0.98 & 0.93 & 0.90 & 0.92 & 0.99 & 0.97 \\
\hline lyaa & 0.98 & 0.94 & 0.95 & 0.95 & 0.96 & 0.87 \\
\hline $\mathrm{ma}$ & 0.94 & 0.92 & 0.95 & 0.93 & 0.94 & 0.93 \\
\hline na & 0.92 & 0.91 & 0.93 & 0.93 & 0.92 & 0.91 \\
\hline naa & 0.97 & 0.95 & 0.98 & 0.96 & 0.97 & 0.96 \\
\hline Nya & 0.96 & 0.94 & 0.97 & 0.93 & 0.96 & 0.95 \\
\hline $\mathrm{pa}$ & 0.94 & 0.92 & 0.95 & 0.93 & 0.94 & 0.93 \\
\hline ppa & 0.88 & 0.86 & 0.90 & 0.87 & 0.88 & 0.87 \\
\hline bha & 0.93 & 0.91 & 0.94 & 0.92 & 0.93 & 0.92 \\
\hline $\mathrm{ra}$ & 0.94 & 0.92 & 0.95 & 0.93 & 0.94 & 0.93 \\
\hline $\mathrm{sa}$ & 0.99 & 0.95 & 0.90 & 0.94 & 0.99 & 0.98 \\
\hline sha & 0.93 & 0.92 & 0.94 & 0.91 & 0.93 & 0.92 \\
\hline sree & 0.94 & 0.91 & 0.97 & 0.92 & 0.95 & 0.93 \\
\hline sya & 0.98 & 0.97 & 0.91 & 0.96 & 0.97 & 0.98 \\
\hline tha & 0.94 & 0.90 & 0.96 & 0.91 & 0.95 & 0.94 \\
\hline the & 0.96 & 0.91 & 0.98 & 0.90 & 0.97 & 0.96 \\
\hline vaa & 0.97 & 0.96 & 0.94 & 0.96 & 0.98 & 0.97 \\
\hline yаa & 0.88 & 0.87 & 0.90 & 0.87 & 0.89 & 0.88 \\
\hline Inna & 0.92 & 0.91 & 0.94 & 0.92 & 0.91 & 0.90 \\
\hline
\end{tabular}

The features extracted using GMA were also classified with Support Vector Machine(SVM). A comparative analysis of the recognition efficiency of SVM and ANN is carried out and 

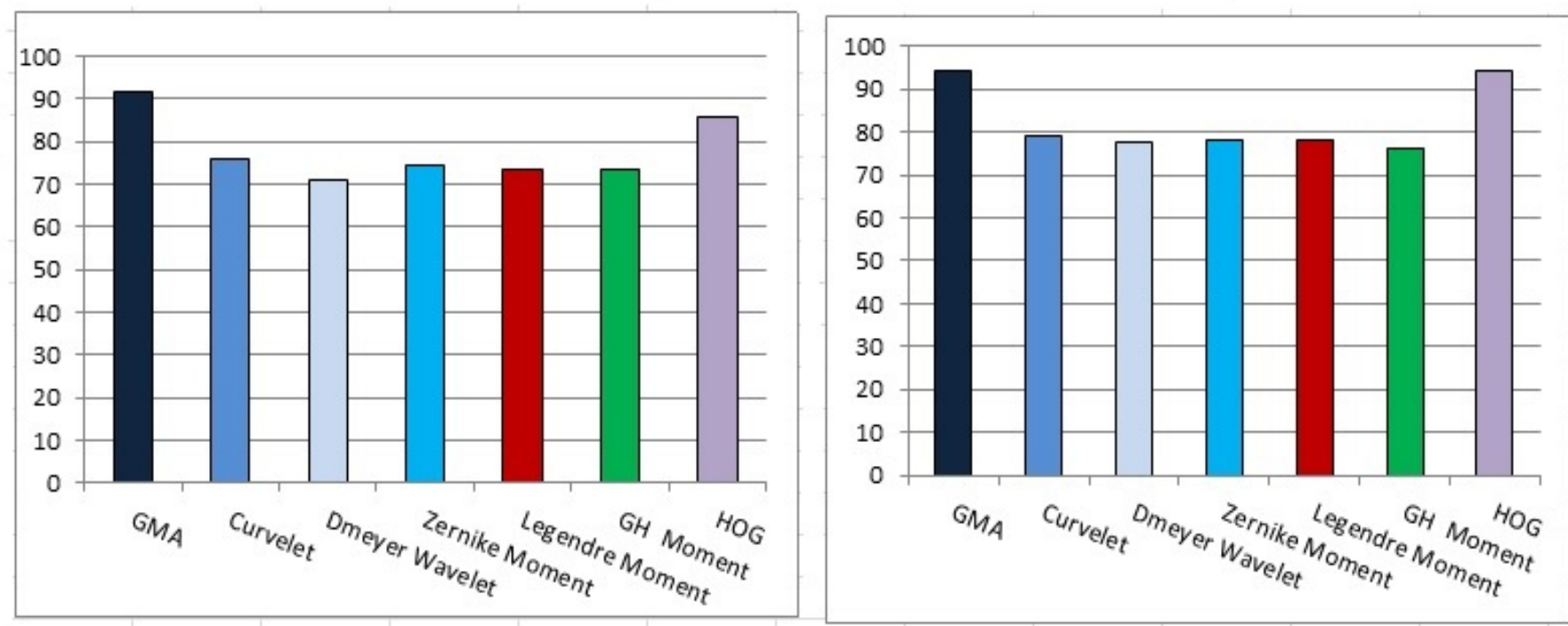

Figure 5: Left:Plot of Recognition efficiency of various methods in Grantha Script with Recognition Efficiency plotted in y-axis and methods in x-axis, Right:Plot of Recognition Efficiency in HPL Dataset with Recognition Efficiency plotted in y axis and methods in $\mathrm{x}$-axis

it is found that, classification using SVM produces higher recognition efficiency compared to ANN(Table 3).

Table 3: Comparison of Recognition Efficiency of Grantha Script using SVM and ANN

\begin{tabular}{lll}
\hline Classiifer & Grantha Dataset & HPL dataset \\
\hline ANN & 91.63 & 94.4 \\
SVM & 92 & 94.7 \\
\hline
\end{tabular}

\section{CONCLUSION}

GMA based feature extraction method gives the highest recognition efficiency in both ancient Grantha characters and standard HPL dataset compared to existing feature extraction methods like Discrete Meyer wavelet, Zernike moment, Legendre moment, Curvelet, Gaussian Hermite moment and HOG.GMA processes images without applying any preprocessing while in other feature extraction methods the images are subjected to binarization before applying the feature extraction methods. HOG method produces recognition efficiency of 94.3 percentage in binary images of HPL dataset but produces recognition efficiency of only 85.8 percentage in Grantha characters. When ANN is used for classification GMA produces high recognition efficiency of 91.63 percentage in Grantha dataset and 94.4 in standard HPL dataset and when SVM is used for classification produces a recognition efficiency of 92 percentage (with Grantha dataset) and 94.7 percentage (with HPL dataset). This feature extraction method if extended to work in other image domains may produce remarkable results.

\section{REFERENCES}

[1] Yann LeCun, Yoshua Bengio, Geoffrey Hinton,2015,Deep learning,NATURE,pp.436-444.

[2] Yosinski,Jason,Jeff Clune, Yoshua Bengio and Hod Lipson,2014,"How transferable are features in deep neural networks",Advances in neural information processing system,pp.3320-3328.

[3] Krizhevsky,Alex,ISutskever and Geoffrey E Hinton,2012,'Imagenet classification using deep convolution neural network",Advances in neural information processing systems,pp.1097-1105.

[4] Jyothi.R.L,’https://github.com/jyothianil/Grantha -script-characters"

[5] Itamar Arel, Derek C. Rose, and Thomas P. Karnowski,2018, "Deep Machine Learning-A New Frontier

in Artificial Intelligence Research", COMPUTATIONAL INTELLIGENCE MAGAZINE,pp.13-18.

[6] N.Arica and and F. T. Yarman-Vural ,2001,"An overview of character recognition focused on off-line handwriting",IEEE Trans on Systems, Man, and Cybernetics,pp.216-233.

[7] Meenu Mohan and R L Jyothi,2015, "Handwritten Character Recognition: A Comprehensive Review on Geometrical Analysis", IOSR Journal of Computer Engineering (IOSR-JCE),pp.83-88.

[8] Ahmed Lawgali, "A survey on Arabic character Recognition", 2015, International Journal of Signal Processing,Imageprocessing and Pattern Recogniton,pp.401-426. 
[9] L.Renjini and and R L Jyothi,2015, "Wavelet based image analysis:A Comprehensive Survey", International Journal of Computer Trends and Technology(IJCTT),pp.134-140.

[10] R.S Suryanath and Anilkumar A,2015,'Study on multiscale image analysis: Theory and applications",International Journal of Computer Trends and Technology(IJCTT),pp.5-10.

[11] Line Eikvil ,2001,'Optical Character Recognition”, citeseer.ist.psu.edu 142042.html,pp.216-233.

[12] Simon Xlian and Qin Lu,1997, "A study of moment functions and its use in Chinese Character Recognition", Proceedings of the Fourth International Conference on Document Analysis and Recognition,pp.572-575.

[13] Aliriza Khotanzard and Yaw Hua Hong,1990,'Invariant Image recogntion by Zernike moments",IEEE transactions on Pattern Analysis and Machine Intellegence,pp.489-497.

[14] Seyed Jabbar Hosaini,Shahpor Alirizance,Majid Ahmadi and Seyed Vahah-Al Din Makki,2013,'Comparison of Legendre,Zernike and Pseudo Zernike Moments for feature extraction in Iris Recognition",IEEE Proceedings of Fifth International Conference on Computational Intellengence and Communication Networks,pp.225-228.

[15] Sajad Farokhi,Siti Mariyam Shamsuddin,U.U Sheikh and Jan Flusser,2014,'Near Infrared Face Recognition Comparison of Moment based approaches",Near Infrared Face Recognition Comparison of Moment based approaches,pp.129-135.

[16] Sajad Farokhi,Siti Mariyam Shamsuddin,U.U Sheikh and Jan Flusser,2013,'Face Recognition using Moments and wavelets", International Journal of Engineering Research and Applications(IJERA),pp.82-95.

[17] Dinggang Shen and Horacce H.S, 1999, "Discriminative wavelet shape descriptors for recognition of 2D patterns", journal of Pattern Recognition Society,pp.151-165.

[18] R.Ahila Priyadharshini,S.Arivazhagam and S.Jeypria,2012,'Object Recognition with Wavelet-Based Salient Points",Global Trands in Information Systems and Software Applicatons Springer,pp.532-541.

[19] Bo Yang,Jitka Kostkov,Jan Flusser and Tomas Suk,2017,"Scale in variants from Gaussian Hermite Moments",Signal processing Elsevier,pp.77-84.

[20] Anca Apatran, Alexandrina Rauthor ogozan, Simina Emerich and Abdelziz Bensrhau,2018, "Wavelets as features for Object Recogntion", Acta Tehnica Napocensis -Electronics and Telecommunications,pp.23-26.

[21] Juilong Zhang,Yinghui Wang ,Zhiyu Zhang and Chunti Xia,2011,'Comparison of wavelet ,Gabor and curvelet transform for Face recognition",Optica Application,pp.183-193.
[22] Angshul Majumdar and Arusharka Bhatacharya, 2009, "A Comparative study in wavelets, Curvelet and Contourlets as feature sets for Pattern Recognition",International Arab journal of Information Technology,pp.936-938.

[23] R L Jyothi and M Abdul Rahiman,2017, "Comparative Analysis of Wavelet Transforms in the Recognition of Ancient Grantha Script",International Journal of Computer Theory and Engineering,pp.235-241.

[24] Kai-ping Feng,Fang Yuan,2013, "Static Gesture Recognition based on HOG characters and Support Vector machines",2nd International Symposium on Instrumentation and Measurement, Sensor Network and Automation (IMSNA) IEEE ,pp.47-51. 